\title{
Language network reorganization before and after temporal lobe epilepsy surgery
}

\author{
Olivia Foesleitner, MD, ${ }^{1}$ Benjamin Sigl, MD, ${ }^{1}$ Victor Schmidbauer, ${ }^{1}$ Karl-Heinz Nenning, MSc, ${ }^{1}$ \\ Ekaterina Pataraia, MD, ${ }^{2}$ Lisa Bartha-Doering, PhD, ${ }^{3}$ Christoph Baumgartner, MD, ${ }^{4}$ \\ Susanne Pirker, MD, ${ }^{4}$ Doris Moser, MSc, PhD, ${ }^{2}$ Michelle Schwarz, ${ }^{2}$ \\ Johannes A. Hainfellner, MD, ${ }^{5}$ Thomas Czech, MD, ${ }^{6}$ Christian Dorfer, MD, ${ }^{6}$ Georg Langs, PhD, ${ }^{1}$ \\ Daniela Prayer, MD, ${ }^{1}$ Silvia Bonelli, MD, PhD, ${ }^{2}$ and Gregor Kasprian, MD ${ }^{1}$
}

Departments of ${ }^{1}$ Biomedical Imaging and Image-guided Therapy, ${ }^{2}$ Neurology, and ${ }^{3}$ Pediatrics and Adolescent Medicine, Medical University of Vienna; ${ }^{4}$ General Hospital Hietzing with Neurological Center Rosenhuegel, Vienna; and ${ }^{5}$ Institute of Neurology and ${ }^{6}$ Department of Neurosurgery, Medical University of Vienna, Austria

\begin{abstract}
OBJECTIVE Epilepsy surgery is the recommended treatment option for patients with drug-resistant temporal lobe epilepsy (TLE). This method offers a good chance of seizure freedom but carries a considerable risk of postoperative language impairment. The extremely variable neurocognitive profiles in surgical epilepsy patients cannot be fully explained by extent of resection, fiber integrity, or current task-based functional MRI (fMRI). In this study, the authors aimed to investigate pathology- and surgery-triggered language organization in TLE by using fMRI activation and network analysis as well as considering structural and neuropsychological measures.
\end{abstract}

METHODS Twenty-eight patients with unilateral TLE (16 right, 12 left) underwent T1-weighted imaging, diffusion tensor imaging, and task-based language fMRI pre- and postoperatively $(n=15$ anterior temporal lobectomy, $n=11$ selective amygdalohippocampectomy, $n=2$ focal resection). Twenty-two healthy subjects served as the control cohort. Functional connectivity, activation maps, and laterality indices for language dominance were analyzed from fMRI data. Postoperative fractional anisotropy values of 7 major tracts were calculated. Naming, semantic, and phonematic verbal fluency scores before and after surgery were correlated with imaging parameters.

RESULTS fMRI network analysis revealed widespread, bihemispheric alterations in language architecture that were not captured by activation analysis. These network changes were found preoperatively and proceeded after surgery with characteristic patterns in the left and right TLEs. Ipsilesional fronto-temporal connectivity decreased in both left and right TLE. In left TLE specifically, preoperative atypical language dominance predicted better postoperative verbal fluency and naming function. In right TLE, left frontal language dominance correlated with good semantic verbal fluency before and after surgery, and left fronto-temporal language laterality predicted good naming outcome. Ongoing seizures after surgery (Engel classes ID-IV) were associated with naming deterioration irrespective of seizure side. Functional findings were not explained by the extent of resection or integrity of major white matter tracts.

CONCLUSIONS Functional connectivity analysis contributes unique insight into bihemispheric remodeling processes of language networks after epilepsy surgery, with characteristic findings in left and right TLE. Presurgical contralateral language recruitment is associated with better postsurgical language outcome in left and right TLE.

https://thejns.org/doi/abs/10.3171/2020.4.JNS193401

KEYWORDS temporal lobe epilepsy; epilepsy surgery; fMRI; functional connectivity; language networks

$\mathrm{E}$ PILEPSY surgery is the recommended treatment option in drug-resistant temporal lobe epilepsy (TLE) and offers an up to $80 \%$ chance of seizure freedom. ${ }^{1-4}$ Uncontrolled seizures are a known risk factor for progressive cognitive impairment, with language and memory functions being most frequently affected. ${ }^{5,6}$ Timely evaluation for epilepsy surgery is thus recommended. ${ }^{3}$

Epilepsy surgery itself can entail language deficits, particularly but not only in left-sided TLE. ' However, a few patients even improve in some cognitive domains

ABBREVIATIONS ATL = anterior temporal lobectomy; DTI = diffusion tensor imaging; FA = fractional anisotropy; $F C=$ functional connectivity; fMRI = functional $M R I ; I F G$ $=$ inferior frontal gyrus; $\mathrm{LI}=$ laterality index; $\mathrm{p}$ uncorr = uncorrected $\mathrm{p}$ value; $\mathrm{ROI}=$ region of interest; $\mathrm{SAH}=$ selective amygdalohippocampectomy; TLE = temporal lobe epilepsy; T1WI = T1-weighted imaging.

SUBMITTED December 16, 2019. ACCEPTED April 7, 2020.

INCLUDE WHEN CITING Published online July 3, 2020; DOI: 10.3171/2020.4.JNS193401. 
postoperatively. ${ }^{7}$ As opposed to classic anterior temporal lobectomy (ATL), selective amygdalohippocampectomy (SAH) spares presumably functioning neocortical tissue and should thereby reduce morbidity. However, this protective effect on cognitive outcome is not statistically significant, ${ }^{8-10}$ and the extent of surgery is thus not a precise prognostic marker.

Diffusion tensor imaging (DTI) measures structural brain connectivity ${ }^{11}$ and has repeatedly revealed widespread decreases in several major tracts after TLE surgery, with ipsilesional predominance..$^{2}$ Increases of white matter integrity as an indicator of neuroplasticity, however, are inconsistently found. ${ }^{12-14}$ Thus, structural imaging alone fails to fully explain the variable neuropsychological profiles in surgical TLE patients.

Functional MRI (fMRI) is widely used for noninvasive presurgical language mapping. Although activation analysis reliably detects task-specific cortical activation used to determine hemispheric dominance, this method does not provide information about the interactions between these task-related regions and therefore does not fully account for the modern language model as a multilayered and bihemispheric network..$^{15}$ fMRI functional connectivity (FC) analysis enables the investigation of these network characteristics and may thus provide a novel perspective that complements language mapping.

In this study of patients with drug-resistant TLE before and after surgery, we investigated language organization associated with cortical language representations (taskbased fMRI activation analysis) and language networks (FC analysis), considering anatomy (type of resection), structural connectivity (DTI), and clinical language function.

\section{Methods}

This study was approved by the local ethics committee and conducted in accordance with the Helsinki Declaration of 1975. All participants gave written informed consent.

\section{Participants}

We retrospectively screened for patients with unilateral TLE who had undergone presurgical task-based language fMRI at our institution and proceeded to partial temporal lobe resection during the period from April 2012 to November 2017. After exclusion of 3 patients due to lack of raw data $(\mathrm{n}=2)$ or insufficient imaging quality $(\mathrm{n}=$ 1), the final study group comprised 28 patients (16 right, 12 left TLE) with postoperative functional scans obtained between March 2016 and September 2018. Twenty-two healthy subjects without history of neurological or psychiatric disease and with no structural alterations on MRI were scanned once using the same imaging protocol.

Diagnosis of unilateral TLE was based on typical seizure semiology and prolonged ictal and interictal videoEEG interpreted by experienced epileptologists. Clinical data were retrieved from the hospital information system or directly from the physicians in charge. All patients had structural imaging with a dedicated 3T MRI epilepsy protocol, including high-resolution sagittal isotropic FLAIR,
T1-weighted imaging (T1WI), axial diffusion- and susceptibility-weighted imaging, T2WI, and an inversion recovery sequence in coronal slices perpendicular to the long axis of the hippocampus, as well as contrast-enhanced T1WI in cases of tumor. Fifteen patients had clear imaging signs of unilateral hippocampal sclerosis (7 left, 8 right) congruent with the clinical origin of the seizure. MRI showed no structural lesions in 9 patients (3 left, 6 right), low-grade tumors in 3 patients (1 left, 2 right), and only gliosis of the inferior temporal gyrus ipsilateral to the seizure origin in 1 patient (patient 8; see Supplementary Table 1 for patients listed by number).

Two neurosurgeons performed ATL in 15 patients (5 left, 10 right), transsylvian SAH in 11 patients (5 left, 6 right), lesionectomy of a calcified lesion in the left fusiform gyrus in 1 patient (patient 10), and selective resection of the left posterior hippocampus and parahippocampal gyrus in 1 patient (patient 9). Histopathological workup of surgical specimens revealed hippocampal sclerosis in 16 patients (7 left, 9 right), focal cortical dysplasia in 5 patients (2 left, 3 right), WHO grade I ganglioglioma in 3 patients (1 left, 2 right), gliosis in 2 patients (2 right), mild malformation of cortical development in 1 patient (1 right), and combined hippocampal sclerosis and heterotopia in 1 patient (1 left). Type of surgery and MRI and histopathologic findings for each patient are outlined in Supplementary Table 1.

Performance scores for the Boston Naming Test ${ }^{16}$ and the Regensburg Verbal Fluency Test ${ }^{17}$ were collected from clinical neuropsychological reports. Ability in naming and phonematic and semantic verbal fluency was categorized as "average" or "impaired" according to standardized cutoff values, i.e., average performance was defined as a score above 56 for naming, and a score within 1 SD for verbal fluency.

\section{Imaging Data Acquisition}

All MRI scans were acquired on the same 3T MRI scanner (Achieva, Philips Healthcare Systems). For fMRI, a gradient-echo echo planar imaging sequence was used, and DTI data were measured with an axial spin-echo echo planar imaging sequence (see Supplementary Methods for acquisition parameters). Pre- and postoperative 1-mm isovoxel T1WI was acquired for anatomical coregistration. Language fMRI consisted of a verb-generation and semantic task in a block design, as described in the Supplementary Methods.

\section{Imaging Data Analysis}

For fMRI activation analysis, standard preprocessing (see Supplementary Methods for details) was followed by first-level analysis according to the general linear model. Only scans with significant task-related activations in the inferior frontal gyrus (IFG) and superior temporal gyrus at an uncorrected $\mathrm{p}$ value ( $\mathrm{p}$ uncorr) threshold $<0.001$ were considered for further analysis. For group analysis, preoperative scans (verb-generation and semantic task) were contrasted to postoperative scans at $\mathrm{p}<0.001$ for left or right TLEs separately.

Additionally, a metric language laterality index (LI) 
TABLE 1. Demographic data of healthy subjects and patients: no significant group differences

\begin{tabular}{|c|c|c|c|c|c|c|}
\hline & \multicolumn{2}{|r|}{ Control Group } & \multicolumn{2}{|r|}{ Lt TLE } & \multicolumn{2}{|r|}{ Rt TLE } \\
\hline & No. & Median (min-max) & No. & Median (min-max) & No. & Median (min-max) \\
\hline \multicolumn{7}{|l|}{ Sex } \\
\hline Males & 14 & & 6 & & 7 & \\
\hline Females & 8 & & 6 & & 9 & \\
\hline \multicolumn{7}{|l|}{ Handedness } \\
\hline $\mathrm{Rt}$ & 21 & & 10 & & 13 & \\
\hline $\mathrm{Lt}$ & 1 & & 2 & & 3 & \\
\hline Age at seizure onset, yrs & & NA & & $22(1-40)$ & & $23(1-48)$ \\
\hline Epilepsy duration, mos & & NA & & $12(3-41)$ & & $10(2-50)$ \\
\hline Age at $1 \mathrm{st}$ MRI, yrs & & $34(23-52)$ & & $34(15-52)$ & & $40(27-54)$ \\
\hline Age at op, yrs & & NA & & $35(15-53)$ & & $40(28-55)$ \\
\hline Time btwn op \& 2nd MRI, mos & & NA & & $6(3-63)$ & & $7(2-50)$ \\
\hline \multicolumn{7}{|l|}{ Op group } \\
\hline SAH & NA & & 6 & & 6 & \\
\hline ATL & NA & & 5 & & 10 & \\
\hline Other & NA & & 2 & & 0 & \\
\hline
\end{tabular}

NA = not applicable.

Values are presented as numbers of subjects for the control group and numbers of patients for the TLE groups unless otherwise indicated.

was calculated from each activation map using a bootstrap algorithm. ${ }^{18}$ Broca's and Wernicke's areas and their contralateral homologs were defined according to the network atlas integrated in the CONN toolbox, congruent to FC analysis. ${ }^{19}$ This parcellation is based on an independent component analysis of 497 subjects from the Human Connectome Project database. In cases of 2 runs per task ( $\mathrm{n}=$ 5 ), the mean value was calculated. For each study participant, the LIs for Broca's and Wernicke's area separately and combined in the verb-generation task, semantic task, and both tasks were determined, resulting in 9 values per subject. This allowed us to independently evaluate language production and comprehension. Metric LI values ranged from -1 (strong right language dominance) to +1 (strong left language dominance). Left language dominance was defined as $L I \geq 0.2$, right-sided dominance accordingly as $\mathrm{LI} \leq-0.2$, and bilateral language distribution as values between. ${ }^{20}$

For FC analysis, default preprocessing settings were applied in the CONN toolbox version 17. $\mathrm{f}^{19}$ (see Supplementary Methods for details), and correctness was checked visually. All 100 time points per run were included to avoid introducing artificial fluctuations by concatenation of data. ${ }^{21}$ Bivariate correlation was performed for 103 supratentorial regions of interest (ROIs) according to the FSL Harvard-Oxford atlas and for the 4 language network ROIs used for the laterality indices. Correlation coefficients were transformed into Fisher's z scores reflecting connectivity strength and corrected with false discovery rate correction $<0.05$.

Postsurgical DTI data were analyzed with deterministic tractography in IntelliSpace Portal version 10 (Philips Healthcare Systems). The following 7 major tracts were manually visualized by 2 coauthors not blinded to clinical history: uncinate fasciculus, inferior fronto-occipital fas- ciculus, inferior longitudinal fasciculus, fornix, cingulum, arcuate fasciculus, and anterior commissure. Subsequently, fractional anisotropy (FA) ratios of ipsilateral divided by contralateral tracts were calculated as an index of fiber integrity. ${ }^{22}$ Associations with side and type of surgery, time after surgery, seizure outcome, and language scores were investigated.

\section{Statistical Analysis}

All statistical analyses were performed with IBM SPSS Statistics for Macintosh (version 26.0, IBM Corp.). Demographic, clinical, and motion data were tested for group differences and correlated with LI and FC measures. Tukey's test was performed for post hoc analysis, a chisquare test was applied to categorical data, and a nonparametric Mann-Whitney test was performed for subgroup analyses. Differences between pre- and postoperative scans were analyzed with paired t-tests. FA values were analyzed descriptively with scatter plots as well as a nonparametric Mann-Whitney test or Spearman's correlation coefficient.

\section{Results}

\section{Clinical Parameters}

Healthy subjects and left- or right-TLE patients did not differ significantly in demographic parameters. The median interval between surgery and postoperative MRI was 6 months (range 2-63 months). Selected demography is outlined in Table 1. Seizure outcome was Engel class I in 13 patients (6 left, 7 right), class II in 3 patients (left), class III in 3 patients (right), and class IV in 4 patients (1 left, 3 right), and no information was available in 5 patients ( 2 left, 3 right). The median follow-up time was 3.2 
LEFT TLE
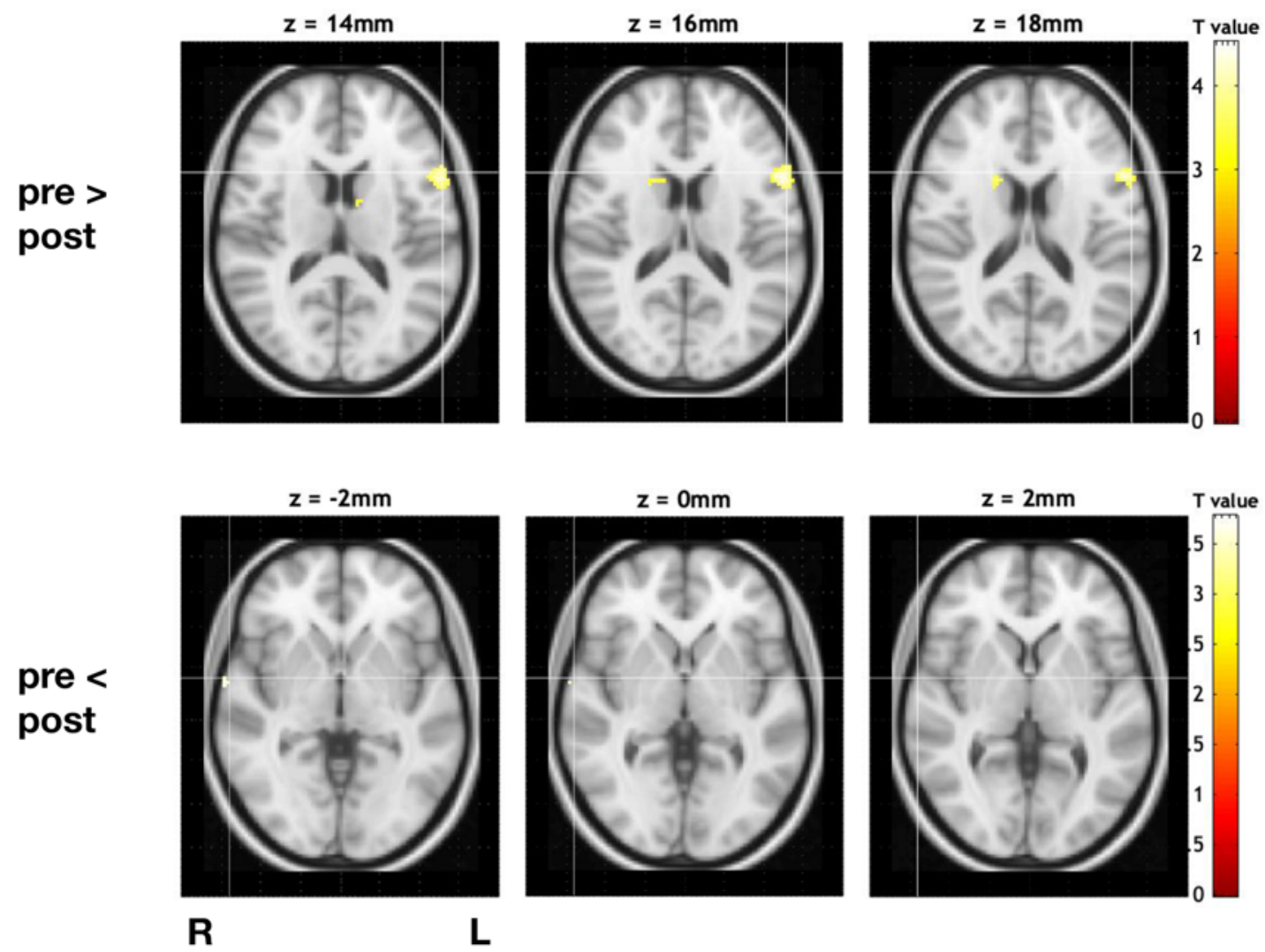

FIG. 1. Activation analysis in left TLE (verb generation and semantic task). After surgery, activation in the left inferior frontal gyrus (Broca's area) decreased (upper), while activation in a small area in the right anterior temporal lobe increased (lower); $p$ uncorr < 0.001 .

years (range 1.4-7.5 years; see Supplementary Table 1). Seizure outcomes did not differ between different types of surgery or between patients who had left and those who had right TLE.

\section{MRI Data Quality}

Lack of significant language-related activations led to exclusion of 16/162 fMRI scans ( 8 pre- and 8 postoperative scans). Motion parameters did not show significant group differences. In 1 patient postsurgical DTI data could not be retrieved and thus were not available for analysis (patient 26).

\section{fMRI Activation Analysis}

After surgery, left-TLE patients showed less task-related activation in the left IFG but stronger activation in a small area in the right anterior temporal lobe ( $\mathrm{p}$ uncorr $<0.001$; Fig. 1). Further, left-TLE patients tended to have more bilateral frontal language dominance in post- versus preoperative LI (Supplementary Table 2). In contrast, right-TLE patients had weaker activations in the right IFG postoperatively but small increased activation in the right dorsal insula and right anterior temporal lobe postoperatively ( $\mathrm{p}$ uncorr $<0.001$; Fig. 2). There were no significant differences in language dominance between fMRI tasks.
Preoperative LI was left-dominant in 25 patients (11 left, 14 right TLE) and bilateral in 3 patients (1 left, 2 right TLE). No patient showed right language dominance. Time after surgery showed neither a clear trend nor a significant correlation with LI values or pre- to postoperative change in language dominance.

\section{FC Analysis}

In global network analysis, both patient groups showed significant differences compared to controls (Fig. 3; for values see Supplementary Table 3). In left-TLE patients, left-to-right mesiotemporal connectivity was lower than in healthy subjects while stronger left temporal to right parietal (supramarginal gyrus) connectivity was found. In right-TLE patients, differences compared with controls were more laterally located, with lower FC between the bilateral frontal and anterior temporal regions but higher FC in the left and right posterior temporal areas. After surgery, differences between patients and controls became further pronounced, with ipsilesional predominance. In left TLE, left fronto-temporal but also left-to-right temporal FC decreased after surgery, while connectivity between left frontal and right posterior temporal regions increased. In right-TLE patients, mainly interhemispheric connectivity decreased after surgery, and increases in FC 


\section{RIGHT TLE}
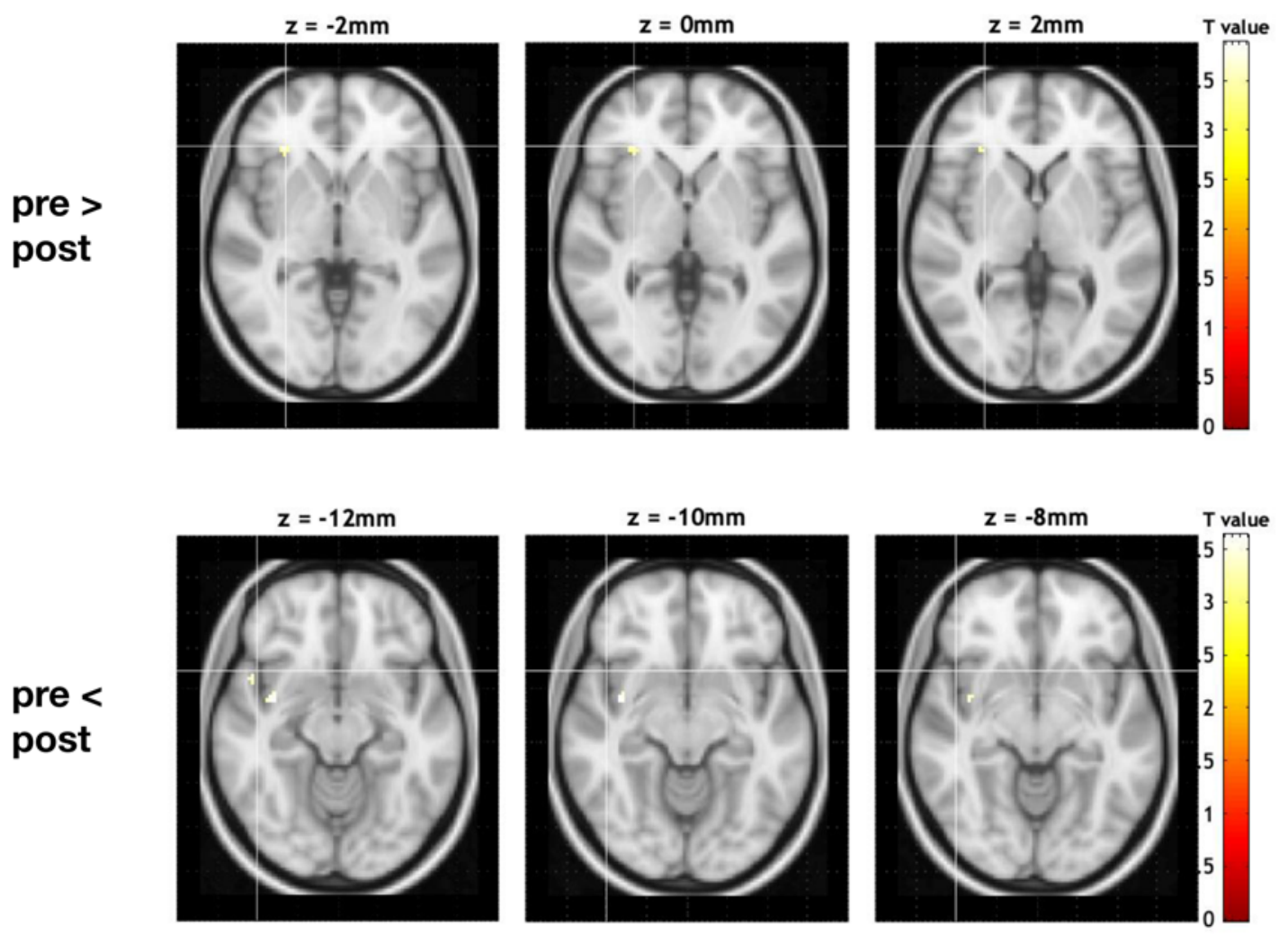

$\mathbf{R}$

FIG. 2. Activation analysis in right TLE (verb generation and semantic task). Postoperatively, task-related activations decreased in the right inferior frontal gyrus (upper) but increased in the right dorsal insula and anterior temporal lobe (lower); p uncorr < 0.001.

were less directed than in left-TLE patients, including several frontal, temporal, and parietal brain regions.

The focused analysis of FC between language ROIs confirmed postoperative decreases in left Broca-to-Wernicke connectivity in left-TLE patients $(\mathrm{p}<0.01)$. Similarly, FC between homotopic right-hemispheric language ROIs was lower in right-TLE patients than in controls before and even more so after surgery $(\mathrm{p}<0.02, \mathrm{p}<0.001$, and $\mathrm{p}=0.02$, respectively). In both TLE groups, left-toright frontal FC decreased after surgery (left $\mathrm{p}<0.01$; right $\mathrm{p}<0.001)$. Time after surgery, however, was not significantly correlated with FC metrics or changes in FC.

\section{DTI Analysis}

FA values in the ipsilesional arcuate fascicle were significantly higher in left- than right-TLE patients $(\mathrm{p}<$ 0.001 ). There was a moderate negative correlation between FA values in the fornix and time after surgery, i.e., lower ipsilesional FA values with longer time after surgery (Spearman's coefficient $0.45, \mathrm{p}=0.03$, linear regression $\mathrm{R}^{2}$ $=0.322)$. In most patients (7/12 left, 16/16 right) the uncinate fasciculus and cingulum were not measurable. FA ratios are given in Supplementary Table 1.

\section{Correlation With Language Function}

Neuropsychological testing was available in $26 / 28$ pa- tients (Supplementary Table 3). There was no significant difference in performance regarding the side of seizure onset other than a trend toward worse naming and phonematic verbal fluency in left-TLE patients.

Postoperative language testing was available in 19/28 patients at 1 time point and in $11 / 28$ patients at 2 time points (median time after surgery 14 months, range 3-37 months; Supplementary Table 3). Within the first 12 postsurgical months, 11 patients were examined and showed the following score dynamics compared to preoperative baseline: naming (stable 6, worse 2 , better 3 ), phonematic fluency (stable 8, worse 2, better 1), and semantic fluency (stable 7 , worse 3 , better 1 ). In 15 patients testing scores at $>12$ months after surgery were available, with the following changes compared to presurgical baseline: naming (stable 11, worse 1, better 3), phonematic fluency (stable 5, worse 3 , better 7), and semantic fluency (stable 13, worse 1 , better 1 ). In summary, naming and semantic verbal fluency tended to stabilize (i.e., return to baseline level) and phonematic fluency even tended to improve, though our data only allowed a conclusion on a group level, as not all patients had 2 postoperative examinations. In patients with more than 1 postsurgical test, the test performed closest to imaging was chosen for comparison with postoperative LI and FC measures (median interval 7 months, range 0-35 months). 


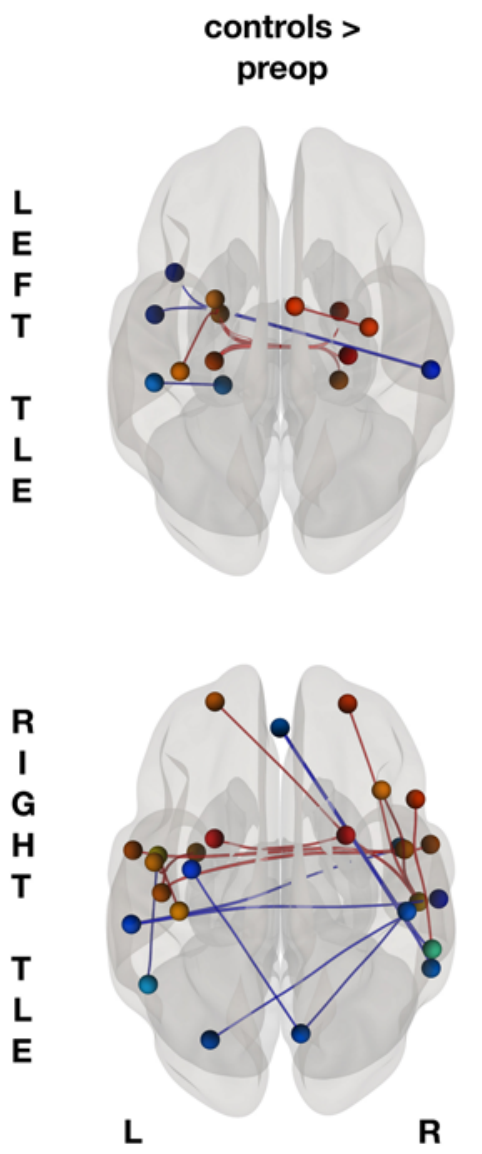

\section{controls > postop}
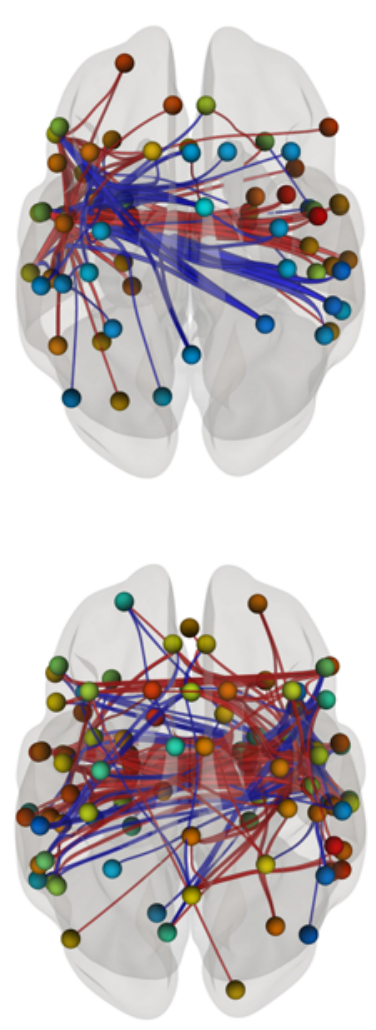

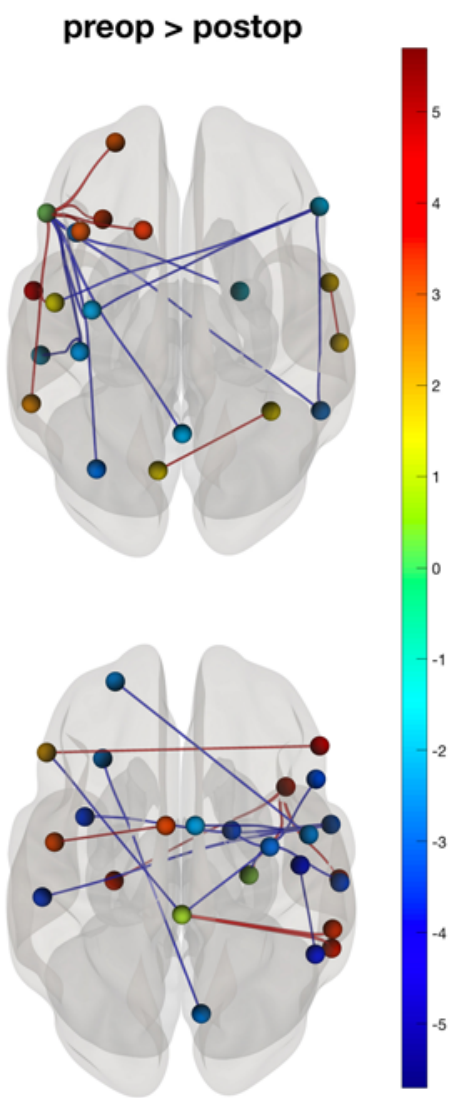

FIG. 3. FC analysis. Preoperatively, left- and right-TLE patients show distinct differences from healthy controls, with more medial FC decreases in left TLE and more lateral FC changes in right TLE. After surgery, bihemispheric FC changes proceed. Pre- to postoperative comparison highlights the left-hemispheric FC decreases and right-hemispheric FC increases in left TLE, while right TLE shows a mixed picture of fronto-temporal FC alterations. Warm colors indicate increases and cold colors indicate decreases in FC (color bar in T-values).

In left-TLE patients, better postoperative semantic verbal fluency and naming function correlated with more atypical fronto-temporal LI prior to surgery $(\mathrm{p}=0.04$ and $\mathrm{p}=0.02$, respectively). In right-TLE patients, on the contrary, better preoperative semantic verbal fluency was associated with left frontal language dominance $(\mathrm{p}=0.02)$ and weaker right fronto-temporal FC $(\mathrm{p}=0.004)$. Moreover, postoperative semantic verbal fluency correlated with more typical frontal LI before surgery in right-TLE patients $(\mathrm{p}=0.005)$. Further, more left-lateralized frontal and temporal LI predicted intact postoperative naming function in right-TLE patients $(\mathrm{p}=0.005)$.

Postsurgical naming function was worse in patients with ongoing seizures (i.e., Engel classes ID-IV) irrespective of TLE side $(p=0.02)$, but not between subgroups preoperatively.

\section{Discussion}

\section{Key Findings}

The principal aim of this study was to use functional and structural imaging to investigate the neural architecture of language function in drug-resistant TLE before and after surgery. We found widespread, predominantly ipsile- sional connectivity decreases before surgery, which were further pronounced after surgery. In left TLE, increased preoperative involvement of homotopic right fronto-temporal language areas was a good prognostic factor for naming and semantic verbal fluency after surgery. In right TLE, less right fronto-temporal FC and more left language dominance correlated positively with pre- and postoperative naming and semantic verbal fluency scores. Ongoing seizures were significantly linked to progressive naming decline after surgery across all patients. Functional imaging findings were not explained by the extent of resection or by DTI measurements.

\section{Language Organization Before Epilepsy Surgery}

Both focal epilepsies and language organization share network properties spanning both hemispheres. Widespread, chronic structural and functional brain network changes were detected far beyond the seizure onset zone in TLE. ${ }^{23,24}$ For instance, Galovic et al. found focal epilepsy patients, the majority with temporal seizure origin, with evidence of global progressive cortical thinning compared with normally aging people..$^{25}$ This volume decrease was particularly pronounced in the first 5 years after seizure 
onset. Similarly to epilepsy, the network concept also applies to language, which is not confined to classical left fronto-temporal regions (i.e., Broca's and Wernicke's areas) but relies on multiple cortical and subcortical structures located in both hemispheres. ${ }^{15}$ Given that most of the healthy population is left language dominant, left-sided seizure onset is associated with particularly severe network alterations and corresponding clinical language deficits. ${ }^{5,726,27}$ Verbal fluency and naming ability are often affected, ${ }^{27}$ which is why we focused on these domains. The concept of wide-range language network disturbances is clearly supported by our findings of bilateral FC changes in TLE patients, with particular ipsilesional but also interhemispheric effects. We confirmed the contralateral contribution of language areas as a good prognostic factor for postoperative language ability seen in previous task-based fMRI studies. ${ }^{28,29}$

Language architecture in right TLE has been less extensively explored, even though deficits are also common. ${ }^{5}$ The supportive role of the right hemisphere regarding language processing was evident in healthy subjects, in whom the right-sided contribution was linked to better language skills. ${ }^{30}$ Further, language deficits may occur in patients after right-sided glioma surgery. ${ }^{31}$ Thus, a strategic lesion in the right hemisphere can measurably impact language function, supported by our findings of widespread network alterations in right TLE. Our study documents for the first time, to our knowledge, a decreased contribution of right fronto-temporal language areas prior to surgery, with further progress after surgery. Decreased right-hemispheric involvement was a good prognostic marker for semantic fluency and naming, in line with the previously published correlation between better verbal fluency and left-lateralized language. ${ }^{29}$ Our findings also strongly suggest that right-TLE patients undergo language network reorganization, but changes in these patients may be more subtle. As the majority of our patients were left language dominant preoperatively, our comparison of left versus right TLE is similar to a comparison of dominant versus nondominant resections.

\section{Language Organization After Epilepsy Surgery}

Epilepsy surgery offers an up to $80 \%$ chance of seizure freedom in drug-resistant TLE and is thus the recommended treatment option. ${ }^{1-4}$ As outlined above, language deficits are commonly attributed to left-sided surgery but can also occur after right-hemispheric resections. ${ }^{26}$ While previous studies have investigated global network changes, we focused on language networks and showed widespread, bilateral remodeling efforts that were even more pronounced after surgery. In a resting-state fMRI study, broad network alterations were found in TLE preoperatively, but only small changes were detected after surgery. ${ }^{32}$ In this study, however, left- and right-TLE patients were not separately analyzed, which might have diluted the results.

In a task-based fMRI study, increased bifrontal activations were found after left-TLE surgery. ${ }^{33}$ This finding is partly confirmed by our study, as the LI in left-TLE patients also tended to become more atypical postoperatively, but mainly due to decreases in left, and less so, increases in right frontal regions (Figs. 1 and 2). In our network analysis, we found decreases in left and increases in right intrahemispheric FC after surgery. Most interestingly, these dynamics were not accompanied by stronger FC between frontal language areas in our sample, as described by Bonelli et al., ${ }^{33}$ but in contrast by a decrease in FC. This finding may indicate not (yet) synchronized left-to-right frontal language processing and could explain why increases in contralateral activation do not necessarily translate into better function. In Bonelli et al., patients with a significant decline in naming test results showed more right middle frontal activations than those with good naming outcomes, a finding indicating that contralateral reorganization is less effective in some patients..$^{33}$ As most of our patients were examined at an early postoperative time point (median 6 months), this low left-to-right frontal connectivity might change and may increase at a later time point. A clear correlation between time after surgery and LI or FC values was not evident in our data, which may be due to statistical power. Language domains in our patients stabilized or even improved on a group level over time, which is commonly seen by neuropsychologists. Such a temporal gradient of reorganization was found in verbal memory after TLE surgery, where activation in the residual posterior left hippocampus was transiently effective at 4 months postoperatively, while the contralateral hippocampus then took over at 12 months. ${ }^{34,35}$ In further longitudinal studies there is a need to explore whether changes in frontal activation and connectivity differ in their temporal evolution and if these translate into language performance.

In order to rule out that our functional findings are merely attributable to structural changes, we considered the extent of resection and integrity of language-related white matter tracts. In particular, the arcuate, uncinate, inferior longitudinal, and inferior frontal occipital fasciculus are known to play important roles in language processing. ${ }^{36}$ As in our sample, higher FA values in the left arcuate fascicle have been reported and indicate functional lateralization. ${ }^{37}$ Previous DTI studies detected widespread, mainly ipsilesional, fiber changes in postoperative TLE that were interpreted as Wallerian degeneration ${ }^{12-14}$ with different patterns of progression over time.$^{38}$ Similarly, we found a moderate association between lower FA values in the ipsilesional fornix and time after surgery. Findings of increases in fiber integrity as an indicator of neuroplasticity are less consistent, i.e., ipsilesional only in left $\mathrm{TLE}^{14}$ or in both left and right TLE. ${ }^{12,13}$ Some authors even hypothesized that these increases in FA were caused by degeneration of crossing fibers, while effective neuroplasticity only occurred in left TLE contralaterally. ${ }^{13}$ Similar to DTI findings, the extent of resection alone does not explain the extremely variable cognitive outcomes after TLE surgery. SAH was proposed as a more protective alternative to classic ATL for sparing the presumably intact lateral temporal cortex, as removal of unaffected tissue evidently deteriorates cognitive outcome. ${ }^{39}$ However, the protective effect of selective surgery was not statistically significant. ${ }^{9,10}$

\section{fMRI Analysis Methods}

Presurgical language fMRI achieves high concordance in language localization compared to invasive alternatives, i.e., Wada testing and direct cortical stimulation. ${ }^{40}$ How- 
ever, fMRI quality depends on task selection, patient cooperation, and statistical interpretation. Further, network properties of language function are dismissed by activation maps. FC analysis could thus complement fMRI language mapping. In activation analysis, changes in blood oxygenation within each voxel are correlated with a specific task design. This method detects cortical functional representations but is blind to their interactions. In contrast, FC analysis correlates signal changes between regions of interest, independent of any task design. High positive correlations are assumed to reflect a functional network. ${ }^{41} \mathrm{FC}$ can be calculated from task-based or resting-state fMRI data, with slight differences, i.e., network strength increases for the function assessed in task-based fMRI. ${ }^{42}$ This difference may be a benefit when only a specific function is of interest, as it increases statistical power, such as language in our case, and spares the need to acquire an additional dataset. In our study, FC turned out to be more sensitive to changes in language organization than activation analysis. However, both tools provide distinct information and should thus be handled as complementary modalities.

Direct cortical stimulation allows precise localization of cortical and subcortical areas essential for a certain function and is the most accurate method for language mapping. However, disadvantages are its invasive character, impact on surgery time and management, and restricted area of possible testing. Presurgical language fMRI is thus often seen as a screening tool for deciding whether awake craniotomy is needed or not. Yet, our study shows the additional unique benefit of fMRI (network) analysis, which allows us to better understand the dynamic, bihemispheric, and network-dependent language architecture.

\section{Limitations}

A few limitations in this study should be considered. Our results are based on group analyses, which do not allow an automatic extrapolation to single subjects. Further, because our inclusion criteria aimed to achieve high group homogeneity at the cost of sample size, we chose nonparametric tests and descriptive analyses whenever needed and possible. Subtle differences in brain connectivity triggered by demographic or clinical features might only become evident in a larger cohort. Neuropsychological data were dichotomized because absolute scores were not consistently documented. This did not allow a more detailed view on score dynamics. There may have been more factors influencing language organization that we could not appreciate, such as medication or education. Extent of resection was only visually analyzed.

\section{Perspectives}

Refining imaging tools to noninvasively assess cognitive networks in epilepsy surgery candidates is important, as current methods fail to explain and predict the full spectrum of cognitive profiles. Structural and functional neuroimaging each contribute unique perspectives on brain functioning, helping neurosurgeons to evaluate possible risks and benefits of surgery. Osipowicz et al. recently tested a trimodal approach with DTI and task-based and resting-state fMRI to predict postsurgical cognitive outcomes in a small cohort of left-TLE patients. ${ }^{43}$ Together with our findings, their study emphasizes the potential of fMRI network analysis. However, further investigations of robust individual analysis methods and the clinical meaning behind connectivity metrics are needed prior to implementation of FC analysis into the clinical routine.

\section{Conclusions}

FC analysis contributes unique insights into the bihemispheric remodeling processes of language networks after epilepsy surgery, with characteristic findings in left and right TLE. Presurgical contralateral language recruitment is associated with better postsurgical language outcomes in left and right TLE.

\section{References}

1. de Tisi J, Bell GS, Peacock JL, et al. The long-term outcome of adult epilepsy surgery, patterns of seizure remission, and relapse: a cohort study. Lancet. 2011;378(9800):1388-1395.

2. Engel J Jr, McDermott MP, Wiebe S, et al. Early surgical therapy for drug-resistant temporal lobe epilepsy: a randomized trial. JAMA. 2012;307(9):922-930.

3. Engel J Jr, Wiebe S, French J, et al. Practice parameter: temporal lobe and localized neocortical resections for epilepsy: report of the Quality Standards Subcommittee of the American Academy of Neurology, in association with the American Epilepsy Society and the American Association of Neurological Surgeons. Neurology. 2003;60(4):538-547.

4. Wiebe S, Blume WT, Girvin JP, Eliasziw M. A randomized, controlled trial of surgery for temporal-lobe epilepsy. $N$ Engl J Med. 2001;345(5):311-318.

5. Bartha-Doering L, Trinka E. The interictal language profile in adult epilepsy. Epilepsia. 2014;55(10):1512-1525.

6. Oyegbile TO, Dow C, Jones J, et al. The nature and course of neuropsychological morbidity in chronic temporal lobe epilepsy. Neurology. 2004;62(10):1736-1742.

7. Helmstaedter C. Cognitive outcomes of different surgical approaches in temporal lobe epilepsy. Epileptic Disord. 2013;15(3):221-239.

8. Bartha L, Trinka E, Ortler M, et al. Linguistic deficits following left selective amygdalohippocampectomy: a prospective study. Epilepsy Behav. 2004;5(3):348-357.

9. Boling WW. Surgical considerations of intractable mesial temporal lobe epilepsy. Brain Sci. 2018;8(2):8.

10. Tanriverdi T, Dudley RW, Hasan A, et al. Memory outcome after temporal lobe epilepsy surgery: corticoamygdalohippocampectomy versus selective amygdalohippocampectomy. $J$ Neurosurg. 2010;113(6):1164-1175.

11. Basser PJ, Mattiello J, LeBihan D. MR diffusion tensor spectroscopy and imaging. Biophys J. 1994;66(1):259-267.

12. Winston GP, Stretton J, Sidhu MK, et al. Progressive white matter changes following anterior temporal lobe resection for epilepsy. Neuroimage Clin. 2013;4:190-200.

13. Pustina D, Doucet G, Evans J, et al. Distinct types of white matter changes are observed after anterior temporal lobectomy in epilepsy. PLoS One. 2014;9(8):e104211.

14. Yogarajah M, Focke NK, Bonelli SB, et al. The structural plasticity of white matter networks following anterior temporal lobe resection. Brain. 2010;133(Pt 8):2348-2364.

15. Poeppel D. The neuroanatomic and neurophysiological infrastructure for speech and language. Curr Opin Neurobiol. 2014;28:142-149.

16. Kaplan E, Goodglass H, Weintraub S. Boston Naming Test. BNT; 1983.

17. Aschenbrenner A, Tucha O, Lange K. RWT: Regensburger Wortflüssigkeits-Test. Hogrefe; 2000.

18. Wilke M, Lidzba K. LI-tool: a new toolbox to assess lat- 
eralization in functional MR-data. J Neurosci Methods. 2007;163(1):128-136.

19. Whitfield-Gabrieli S, Nieto-Castanon A. Conn: a functional connectivity toolbox for correlated and anticorrelated brain networks. Brain Connect. 2012;2(3):125-141.

20. Seghier ML. Laterality index in functional MRI: methodological issues. Magn Reson Imaging. 2008;26(5):594-601.

21. Ganger S, Hahn A, Küblböck M, et al. Comparison of continuously acquired resting state and extracted analogues from active tasks. Hum Brain Mapp. 2015;36(10):4053-4063.

22. Alexander AL, Hurley SA, Samsonov AA, et al. Characterization of cerebral white matter properties using quantitative magnetic resonance imaging stains. Brain Connect. 2011;1(6):423-446.

23. Cataldi M, Avoli M, de Villers-Sidani E. Resting state networks in temporal lobe epilepsy. Epilepsia. 2013; 54(12):2048-2059.

24. Englot DJ, Konrad PE, Morgan VL. Regional and global connectivity disturbances in focal epilepsy, related neurocognitive sequelae, and potential mechanistic underpinnings. Epilepsia. 2016;57(10):1546-1557.

25. Galovic M, van Dooren VQH, Postma T, et al. Progressive cortical thinning in patients with focal epilepsy. JAMA Neurol. 2019;76(10):1230-1239.

26. Drane DL, Pedersen NP. Knowledge of language function and underlying neural networks gained from focal seizures and epilepsy surgery. Brain Lang. 2019;189:20-33.

27. Hamberger MJ, Cole J. Language organization and reorganization in epilepsy. Neuropsychol Rev. 2011;21(3):240-251.

28. Chang YA, Kemmotsu N, Leyden KM, et al. Multimodal imaging of language reorganization in patients with left temporal lobe epilepsy. Brain Lang. 2017;170:82-92.

29. Rosazza C, Ghielmetti F, Minati L, et al. Preoperative language lateralization in temporal lobe epilepsy (TLE) predicts peri-ictal, pre- and post-operative language performance: an fMRI study. Neuroimage Clin. 2013;3:73-83.

30. van Ettinger-Veenstra HM, Ragnehed M, Hällgren M, et al. Right-hemispheric brain activation correlates to language performance. Neuroimage. 2010;49(4):3481-3488.

31. Vilasboas T, Herbet G, Duffau H. Challenging the myth of right nondominant hemisphere: lessons from corticosubcortical stimulation mapping in awake surgery and surgical implications. World Neurosurg. 2017;103:449-456.

32. Maccotta L, Lopez MA, Adeyemo B, et al. Postoperative seizure freedom does not normalize altered connectivity in temporal lobe epilepsy. Epilepsia. 2017;58(11):1842-1851.

33. Bonelli SB, Thompson PJ, Yogarajah M, et al. Imaging language networks before and after anterior temporal lobe resection: results of a longitudinal fMRI study. Epilepsia. 2012;53(4):639-650.

34. Bonelli SB, Thompson PJ, Yogarajah M, et al. Memory reorganization following anterior temporal lobe resection: a longitudinal functional MRI study. Brain. 2013;136(Pt 6):1889-1900.

35. Sidhu MK, Stretton J, Winston GP, et al. Memory network plasticity after temporal lobe resection: a longitudinal functional imaging study. Brain. 2016;139(Pt 2):415-430.

36. Leyden KM, Kucukboyaci NE, Puckett OK, et al. What does diffusion tensor imaging (DTI) tell us about cognitive networks in temporal lobe epilepsy? Quant Imaging Med Surg. 2015;5(2):247-263.

37. Catani M, Allin MP, Husain M, et al. Symmetries in human brain language pathways correlate with verbal recall. Proc Natl Acad Sci U S A. 2007;104(43):17163-17168.

38. Li W, An D, Tong X, et al. Different patterns of white matter changes after successful surgery of mesial temporal lobe epilepsy. Neuroimage Clin. 2019;21:101631.

39. Helmstaedter C, Petzold I, Bien CG. The cognitive consequence of resecting nonlesional tissues in epilepsy surgery- results from MRI- and histopathology-negative patients with temporal lobe epilepsy. Epilepsia. 2011;52(8):1402-1408.

40. Weng HH, Noll KR, Johnson JM, et al. Accuracy of presurgical functional MR imaging for language mapping of brain tumors: a systematic review and meta-analysis. Radiology. 2018;286(2):512-523.

41. Biswal B, Yetkin FZ, Haughton VM, Hyde JS. Functional connectivity in the motor cortex of resting human brain using echo-planar MRI. Magn Reson Med. 1995;34(4):537-541.

42. Fox MD, Raichle ME. Spontaneous fluctuations in brain activity observed with functional magnetic resonance imaging. Nat Rev Neurosci. 2007;8(9):700-711.

43. Osipowicz K, Sperling MR, Sharan AD, Tracy JI. Functional MRI, resting state fMRI, and DTI for predicting verbal fluency outcome following resective surgery for temporal lobe epilepsy. J Neurosurg. 2016;124(4):929-937.

\section{Disclosures}

The authors report no conflict of interest concerning the materials or methods used in this study or the findings specified in this paper.

\section{Author Contributions}

Conception and design: Kasprian, Foesleitner, Schmidbauer, Bartha-Doering, Schwarz, Prayer, Bonelli. Acquisition of data: Kasprian, Foesleitner, Schmidbauer, Pataraia, Baumgartner, Pirker, Moser, Schwarz, Hainfellner, Czech, Dorfer, Langs, Prayer, Bonelli. Analysis and interpretation of data: Kasprian, Foesleitner, Sigl, Nenning, Bartha-Doering, Hainfellner, Czech, Prayer, Bonelli. Drafting the article: Kasprian, Foesleitner. Critically revising the article: Kasprian, Foesleitner, Schmidbauer, Nenning, Bartha-Doering, Schwarz, Dorfer, Prayer, Bonelli. Reviewed submitted version of manuscript: all authors. Approved the final version of the manuscript on behalf of all authors: Kasprian. Statistical analysis: Foesleitner, Nenning. Administrative/ technical/material support: Kasprian, Foesleitner, Sigl, Schmidbauer, Nenning, Pataraia, Baumgartner, Pirker, Moser, Schwarz, Hainfellner, Czech, Dorfer, Langs, Prayer, Bonelli. Study supervision: Kasprian, Foesleitner, Pataraia, Prayer, Bonelli.

\section{Supplemental Information \\ Online-Only Content}

Supplemental material is available with the online version of the article.

Supplementary Methods and Tables. https://thejns.org/doi/ suppl/10.3171/2020.4.JNS193401.

\section{Previous Presentations}

Preliminary results of this work have been previously presented as follows. Oral Presentations: 103rd Annual Meeting of the Radiological Society of North America, 2017, Chicago, Illinois; 52nd Annual Meeting of the German Society of Neuroradiology, 2017, Cologne, Germany; 55th Annual Meeting of the American Society of Neuroradiology, 2017, Long Beach, California; European Congress of Radiology, 2017, Vienna, Austria. Poster Presentations: Brain Plasticity in Epilepsy, 2017, Leuven, Belgium; 14th Annual Meeting of the Austrian Society of Neurology, 2017, Vienna, Austria; 2nd Alpine Chapter Symposium and 16th Austrian fMRI Symposium, 2016, Salzburg, Austria.

\section{Correspondence}

Gregor Kasprian: Medical University of Vienna, Austria. gregor. kasprian@meduniwien.ac.at. 